\title{
Stellenwert und Funktion von Ayahuasca: Ein Vergleich der Anwendungen durch Schamanen und Kirchen
}

\author{
Sylvia Linhart \\ Wien, Österreich
}

\author{
Schlüsselwörter \\ Banisteriopsis caapi - Psychotria viridis . Chacruna . \\ Psychoaktive Pflanzen · Dimethyltryptamin/DMT · Harmin · Harmalin . \\ Serotonin · Depression · Suchtkrankheit · Schamanismus . \\ Santo Daime $\cdot$ Sakrament $\cdot$ Religion
}

\section{Zusammenfassung}

Ayahuasca wurde schon in präkolumbianischer Zeit eingenommen, als indigene Völker die psychoaktive Wirkung des Tranks auf Körper und Geist entdeckten. Hergestellt aus zwei im Amazonas-Regenwald wachsenden Pflanzen, der Liane Banisteriopsis caapi und Blättern von Psychotria viridis, wird Ayahuasca bis heute nicht nur von Schamanen am Amazonas für Heilrituale und schamanische Erfahrungen, sondern auch von kirchlichen Bewegungen Brasiliens als Sakrament eingesetzt. In den letzten Jahren gab Ayahuasca wegen seiner heilenden Wirkung immer häufiger Anlass zu medizinischer und ethnobotanischer Diskussion und Forschung. Die vorliegende Literatur- und Feldstudie beschäftigt sich mit den beiden Haupteinsatzbereichen von Ayahuasca: dem rituellen Gebrauch bei Schamanen und dem Sakrament in christlichen Kirchen. Anwendungsformen des Tranks werden beschrieben und Unterschiede aufgezeigt. Da immer mehr Menschen westlicher Kulturen Kenntnis von und Zugang zu dieser «Medizin» des Amazonas-Regenwalds erlangten, bildet die Frage, ob Ayahuasca ein geeignetes Heilmittel für spezifische Leiden und westliche Zivilisationskrankheiten sein kann, einen weiteren Schwerpunkt der Untersuchung. Bereits heute wird Ayahuasca erfolgreich im medizinisch-therapeutischen Bereich bei Depressionen und Suchtabhängigkeit eingesetzt. Darüber hinaus belegen klinische Studien, dass die dauerhafte, längerfristige Einnahme des Tranks nicht gesundheitsschädlich oder giftig ist und auch nicht abhängig macht. Es konnte sogar ein dauerhafter, positiver Einfluss auf die physische und mentale Gesamtverfassung des Menschen beobachtet werden. Vor dem Hintergrund dieser Erkenntnisse bedarf es noch konsequenter Aufklärung und Erarbeitung sinnvoller gesetzlicher Regelungen sowie internationaler Zulassungen, damit Ayahuasca künftig leichter Heilungssuchenden zugänglich gemacht werden kann.

Überarbeitete und aktualisierte Version einer Projektarbeit im Rahmen des CAS «Ethnobotanik und Ethnomedizin» an der Universität Zürich, Schweiz.

\section{Keywords}

Banisteriopsis caapi · Psychotria viridis · Chacruna .

Psychoactive plants · Dimethyltryptamine/DMT · Harmine - Harmaline . Serotonin · Depression · Addiction · Shamanism · Santo Daime · Sacrament $\cdot$ Religion

\section{Summary}

Significance and Function of Ayahuasca: A Comparison of the Utilizations by Shamans and Churches

Ayahuasca was already taken in pre-Columbian times, when indigenous peoples discovered its psychoactive effects on body and mind. Made of two plants growing in the Amazon rainforest, the liana Banisteriopsis caapi and leaves of Psychotria viridis, the beverage is still used nowadays by Amazonian shamans for healing rituals and shamanic experience as well as by ecclesial movements in Brazil as a sacrament. Due to stated healing effects in recent years Ayahuasca stimulates more and more medical and ethnobotanical discussions and research. This literature and field study deals with two principal areas of application of Ayahuasca: the ritual use by shamans and the use as sacrament in Christian churches. In this paper various applications of Ayahuasca and their differences are shown. Furthermore, due to the increasing number of Western people who got knowledge of and access to the natural medicine from the Amazon rainforest, the question remains whether Ayahuasca could be an appropriate remedy for specific ailments and diseases of the Western civilization. Nowadays, Ayahuasca is being successfully used for the medical treatment of depression and addiction. Moreover, clinical studies have documented that the long-term use of the beverage is neither harmful nor toxic and is not addictive. The investigations furthermore demonstrated a lasting positive influence on the whole physical and mental constitution. In light of these insights, there is still a need for persistent information, useful rules and regulations as well as international licensing in order to make Ayahuasca more easily accessible to all those in search of healing.

\section{KARGER}

Fax +497614520714 Information@Karger.com www.karger.com
() 2015 S. Karger GmbH, Freiburg

$1015-0684 / 15 / 0273-0169 \$ 39.50 / 0$
Sylvia Linhart

Lichtenauergasse 7/29, 1020 Wien, Österreich

sylvia.linhart@freuland.at 


\section{Einleitung}

Die vorliegende Literatur- und Feldstudie zeigt die beiden Haupteinsatzbereiche von Ayahuasca - einerseits bei Schamanen im Amazonasgebiet, anderseits in brasilianischen Kirchen - und die Unterschiede in deren Anwendung auf. Dabei wird ein Hauptaugenmerk auf den Stellenwert von Ayahuasca im Leben dieser Anwender und Kulturen gelegt und ein weiterer Schwerpunkt auf die Frage gesetzt, ob diese Medizin des südamerikanischen Regenwalds ein geeignetes Heilmittel für spezifische Leiden, Krankheiten und Missverhältnisse im menschlichen Körper auch im europäischen Raum sein kann.

\section{Was ist Ayahuasca?}

Betrachtet man vorerst die rein stofflich-materielle Seite, so handelt es sich um einen Trank, der aus einer Mischung von hauptsächlich zwei Pflanzen hergestellt wird: aus der Liane Banisteriopsis caapi (Spruce ex Griseb.) Morton (Abb. 1) aus der Familie der Malpighiaceae (Malpighiengewächse) und den Chacruna-Blättern Psychotria viridis Ruiz \& Pav. (Abb. 2) aus der Familie der Rubiaceae (Rötegewächse). In beiden Fällen handelt es sich um Pflanzen, die im tropischen Südamerika (Amazonas und Orinoco-Gebiet, Ecuador, Kolumbien, Peru) beheimatet sind [1-9].

Sowohl B. caapi als auch $P$. viridis enthalten psychoaktive Alkaloide. Beziehen wir die Wirkung auf den mensch-

Abb. 1. Banisteriopsis caapi.

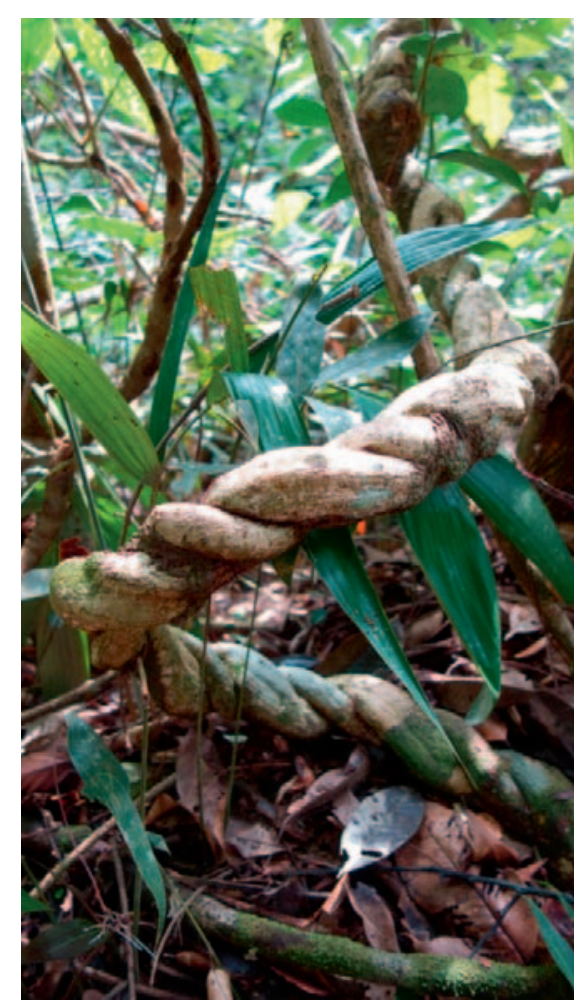

lichen Körper mit ein, so spricht man in unserem Kulturkreis von einem psychoaktiven (bewusstseinsverändernden) Trank, da diese Inhaltsstoffe Wirkungen auf die menschliche Psyche und das Bewusstsein ausüben, die einerseits lähmend, dämpfend sowie eingrenzend und anderseits anregend, stimulierend sowie erweiternd sein können [3, 4, 6]. Pharmakologisch erfolgte im Laufe der Zeit eine Kategorisierung der psychoaktiven Substanzen im Allgemeinen aufgrund der einzelnen Wirkungen in i) Stimulantien, ii) Sedativa, Hypnotika, Narkotika, und iii) Halluzinogene $[6,10]$. Unter Halluzinogene fallen alle Pflanzen oder Substanzen mit beträchtlicher Auswirkung auf die Wahrnehmung von Raum und Zeit oder auf die Auslösung von Emotionen [10].

In der gängigen Literatur wird Ayahuasca unter die Halluzinogene eingereiht. Da der Begriff Halluzinogen jedoch in Zusammenhang mit Halluzination gesehen wird, die per definitionem als Sinnestäuschung mit Realitätscharakter $[6,11]$ beschrieben wird, wird dieser Arbeit der umfassendere Begriff "psychoaktive oder bewusstseinsverändernde» Substanz zugrunde gelegt, da er der Erfassung des Themas mehr Raum gibt.

Beschäftigt man sich mit der kulturellen und kosmologischen Ebene, so ist Ayahuasca für indigene Völker - abgesehen von Medizin - ein heiliger Trunk, ein Pflanzenlehrer und ein Verbindungs-/Kommunikationsmittel zu den Göttern und Ahnen sowie zu den anderen Wirklichkeiten [3, 4, 6-8, 12-14].

In Religionsgemeinschaften wie Santo Daime, União do Vegetal (UDV) und Barquinha ist es Sakrament, Heilmittel, Reinigung und Mittel zur Autoanalyse.

Anhand der eben gezeigten, verschiedenen Erklärungszugänge lässt sich erkennen, dass eine zum heutigen Zeitpunkt abschliessende Definition oder Erklärung, was Ayahuasca ist, nahezu unmöglich ist, da einer wissenschaftlichen Definition schon aufgrund der Begrenztheit der Worte und des Sinns, der ihnen beigemessen wird, sowohl gedanklich als auch sprachlich bedingte Grenzen gesetzt sind. Eine eindeutige Definition oder Erklärung hängt daher von der jeweiligen Perspektive und Forschungsfrage oder vom Interesse des Betrachters ab und beschreibt dann jeweils nur den betrachteten Ausschnitt.

Vielleicht ist genau diese Beschränktheit in der Definitionsgebung der Grund, warum einerseits die genaue Herkunft dieser «Medizin» unbekannt ist und andererseits die

Abb. 2. Psychotria viridis.

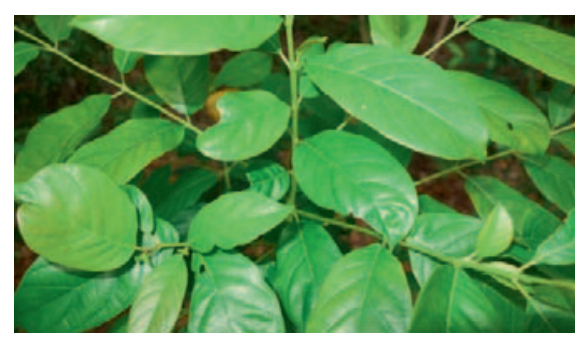


Erforschung von Ayahuasca im Laufe der letzten Jahre zugenommen hat. Lange Zeit gab es zu Ayahuasca nur mündliche Überlieferungen und die Weitergabe des traditionellen Gebrauchs bei indigenen Völkern. Durch das Bekanntwerden des Tranks und seiner positiven Wirkungen auf den menschlichen Körper und die Psyche im Laufe der Geschichte wurde damit auch der westlichen Welt ein weiteres Erforschungsgebiet eröffnet. Botaniker und Mediziner sowie andere Forschungsinteressierte begannen, die Inhaltsstoffe und auch die Wirkungen auf den Menschen genauer zu analysieren. Immer mehr positive Effekte in der Behandlung von suchtabhängigen und depressiven Menschen mithilfe von Ayahuasca konnten beobachtet werden. Dies löste eine Zunahme der Forschung, speziell auch in der Gehirnforschung, aus [15]. Viele Forschungsergebnisse auf der World Ayahuasca Conference vom 25.-27.9.2014 brachten diesen Trend zum Ausdruck (siehe auch Kapitel «Ausblick»).

\section{Geschichte}

Die Literatur spricht davon, dass Ayahuasca bereits seit präkolumbianischer Zeit als psychoaktiver Trank von Schamanen und Medizinmännern am Amazonas für Heilrituale und schamanische Erfahrungen Einsatz findet. Der Gebrauch wurde anscheinend im westlichen Amazonasgebiet (dem heutigen Ecuador) entdeckt $[5,6]$. Bei archäologischen Grabungen in Ecuador wurden Funde gemacht, die als "Hexertöpfe» in die Literatur eingegangen sind. Es handelt sich dabei um einfache, grosse Keramikgefässe, die der Herstellung von Ayahuasca gedient haben und der Milagro-Quevedo-Kultur (500 v. Chr.-1500 n. Chr.) zugerechnet werden [16].

Das Wort Ayahuasca stammt aus dem Quechua und bedeutet «Seelenranke» oder «Geisterliane» $[2-4,6,7]$. Die erste botanische Sammlung der Liane wurde vom Botaniker Richard Spruce (1817-1893) zwischen 1851 und 1854 zusammengetragen. Die originalen Belegexemplare wurden sogar auf Alkaloide hin untersucht. Den ersten Bericht über die psychoaktiven Eigenschaften dieser Liane schrieb M. Villavicencio 1858 in Geografia de la Republica del Ecuador [2]. Seit Spruces Zeit wurde Ayahuasca oft von Reisenden und Forschern erwähnt, aber bislang wenig Beachtung geschenkt. In der Tat wurde Spruces 1851 gesammeltes Material erstmals 1969 chemisch analysiert [3].

Erst in den 1960er-Jahren wurde vom amerikanischen Ethnobotaniker Homer Pinkley bei den Kofán-Indianern Kolumbiens der psychoaktive Gebrauch von P. viridis, und zwar als Ayahuasca-Zusatz, beobachtet und beschrieben. Die Gattung Psychotria wurde botanisch erstmals von Linné beschrieben [6].
Ayahuasca wurde nie von der Inquisition verboten. Erst in den 1950er-Jahren wurde den am Urubamba lebenden Machigengas durch eine Schweizer Mission der Gebrauch von Ayahuasca als Teufelswerk untersagt. Die Unterdrückung des Ayahuasca-Genusses hat daraufhin zu einer Entwurzelung und kulturellen Verwahrlosung geführt [16].

Auch das kulturelle Erbe der Shipibo-Indianer wurde von modernen Missionaren stark zerstört. Die Shipibo hatten eine Art Schrift, die bestimmte Erfahrungen der visionären Welt dekodierte und kommunizierte (Ayahuasca-Muster) [16].

Noch heute setzen verschiedenste indigene Stämme des Amazonas-Gebiets Ayahuasca als Medizin und zu schamanischen Zwecken ein.

Mit der steigenden Verstädterung der ehemaligen Regenwaldgebiete kamen immer mehr Nicht-Indianer mit dem alten Trank in Berührung, was zur Ausbildung eines urbanen Schamanismus führte [6, 9]. Katholische Mestizen sind inzwischen etablierte Stadtschamanen geworden, die den Trank zur Behandlung von Leiden der Städter benutzen. Ihre Rituale sind ein buntes Gemisch aus indianischen und katholischen Gebräuchen. Daneben sind mehrere Ayahuasca-Kirchen sowie ein reger AyahuascaTourismus entstanden [6].

\section{Wirkstoffe und Wirkung}

Die Caapi-Liane (B. caapi) enthält $\beta$-CarbolinAlkaloide wie Harmin, Harmalin, Tetrahydroharman und Harmol als Hauptalkaloide. Daneben finden sich noch weitere verwandte Alkaloide. Die Stängel enthalten an Alkaloiden bis ca. 0,8\%, Zweige bis ca. 0,4\%, Blätter bis ca. $0,7 \%$ und Wurzeln bis ca. 1,9\%. Den grössten Anteil davon bildet Harmin mit 40-96\%. In Stängeln und Rinde kommen auch reichlich Gerbstoffe vor $[2-4,6,10,17]$.

Chacruna-Blätter ( $P$. viridis) enthalten hauptsächlich Dimethyltryptamin (DMT) bis zu ca. $0,6 \%$ und Spuren von 2-Methyltetrahydro- $\beta$-Carbolin $[2-4,6,10]$.

\section{Pharmakologische Wirkung auf den menschlichen Körper}

DMT aus den Chacruna-Blättern ist die eigentlich psychoaktiv wirksame Substanz. Es handelt sich dabei um ein im menschlichen Körper natürlich vorkommendes, von der Hypophyse erzeugtes Hormon. Wird nun DMT zusätzlich zugeführt, so würde dieses sofort vom körpereigenen MAO-A-Enzym (Monoaminooxidase-A-Enzym) abgebaut werden. Allerdings kommt es in Verbindung mit den $\beta$-Carbolin-Alkaloiden der Caapi-Liane, dem zweiten Bestandteil des Tranks, zu einer Hemmung dieses 


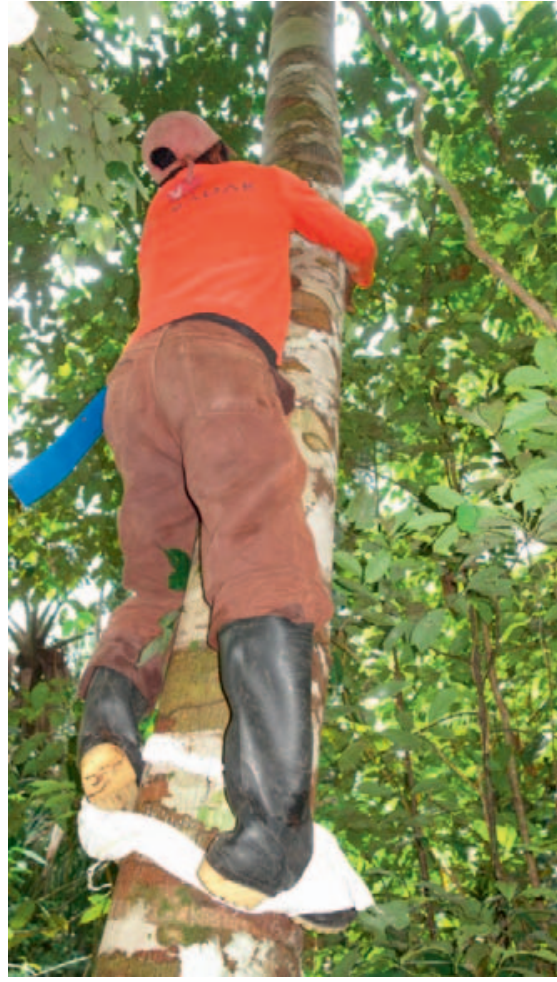

Liane im Rege von Acre, Brasilien.
Abb. 4. Transport der Liane.

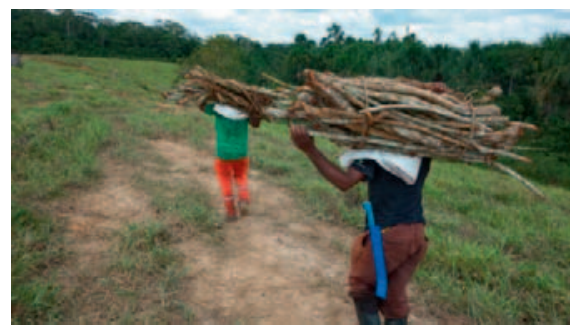

können Zusammenhänge erkannt werden, die noch nie zuvor aufgetaucht sind und der Person Einsichten vermitteln, die sie bis zu diesem Zeitpunkt noch nicht realisieren konnte, da diese durch den verminderten Zugriff auf die speziellen Gehirnregionen noch «undenkbar» waren.

\section{Herstellung von Ayahuasca}

Dieser Abschnitt gibt die Beobachtungen und Resultate meiner Feldforschung im brasilianischen Acre (im Amazonas-Einzugsgebiet an der Grenze zu Peru und Bolivien) - der Geburtsstätte der verschiedenen religiösen Bewegungen, die mit Ayahuasca arbeiten - im Zeitraum vom 4. bis zum 20. Februar 2014 wieder. Im Rahmen einer teilnehmenden Beobachtung konnte ich zwei Männer aus dem Dorf Rodrigues Alves bei der Ernte begleiten und anschliessend an der Zubereitung teilnehmen. Es handelte sich dabei um eine Ernte der Kirche Santo Daime und der Herstellung des Trunks «Daime» für ihre kirchlichen Veranstaltungen.

\section{Ernte}

Die Caapi-Liane aus dem Regenwald wird unter grossen körperlichen Strapazen händisch geerntet (Abb. 3). Lange Fussmärsche in der tropischen Hitze des Regenwalds (Luftfeuchtigkeit bis zu 95\%) bis zu den Plätzen, wo die Lianen wachsen, werden hierfür in Kauf genommen. Der kundige Suchende kennt entweder die bestimmten Plätze oder erkennt diese bereits an der umliegenden Vegetation. Die Liane mit einem Durchmesser von bis zu ca. $10-15 \mathrm{~cm}$ wird mit Macheten von den Bäumen, an denen sie sich hochwinden, heruntergeschnitten. Der Stängel ist sehr wasserhaltig, sodass man von der Schnittstelle direkt das gespeicherte Wasser trinken kann. Dies wird als Erfrischung von den erntenden Personen sofort bei der Ernte genutzt. Nach der Entfernung aus dem Geäst wird die Liane direkt vor Ort in etwa $1 \mathrm{~m}$ lange Stücke zerteilt und diese zu Bündeln zusammengebunden. Die in meiner Anwesenheit geerntete Liane wog 17 $\mathrm{kg}$, wurde zu zwei Bündeln zusammengefasst und von zwei Männern auf ihren Schultern aus dem Regenwald zum ca. 30 Minuten entfernt stehenden Auto transportiert (Abb. 4). 
Abb. 5. Abkochungsprozess.

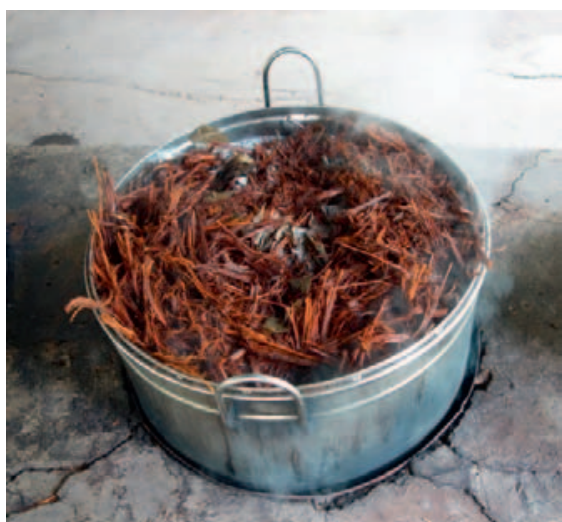

Chacruna-Pflanzen (P. viridis), der zweite Bestandteil zur Herstellung des Ayahuasca-Tranks, werden in vielen Fällen schon in der Nähe der Wohnstätten kultiviert. Da es sich um ein strauchartiges Gewächs handelt, ist die Ernte der Blätter einfacher als jene der Liane.

\section{Vorbereitung - Abkochung - Reduzierung}

Die Caapi-Liane wird in ca. 0,5 cm dicke Fasern zerteilt. Dies geschieht entweder händisch durch Zerklopfen auf Baumstümpfen mit selbst hergestellten Holzschlägeln oder durch einen kleinen Häcksler. Nach den Vorbereitungsarbeiten werden abwechselnd Lianenfasern und ganze Chacruna-Blätter in grossen Kochtöpfen bis obenauf geschichtet und der verbleibende Platz mit Wasser aufgefüllt. Auf Holzfeuern werden diese Töpfe zum Kochen gebracht (Abb. 5), und der erste Kochprozess dauert etliche Stunden, bis das Pflanzenmaterial ausgekocht ist. Die Flüssigkeit, die daraus entsteht, wird aus den einzelnen Töpfen abgegossen und gesammelt. Zwei Männer heben dazu mit einem langen Holzast den Kochtopf an einem Henkel hoch und giessen die heisse Flüssigkeit über eine speziell angefertigte Blechrinne durch ein Baumwollsieb in Eimer (Abb. 6).

Danach wird diese abgegossene Flüssigkeit wieder in bereits mit Pflanzenmaterial kochende Töpfe eingefüllt und weiter gekocht, um eine dickere Konsistenz zu erreichen. Diese Reduktionen werden mehrmals wiederholt. Die genauen Flüssigkeitsmengen und die Anzahl dieser mehrmalig wiederholten Kochvorgänge wurden mir nicht verraten und laufen auch bei jedem dieser Prozesse individuell $\mathrm{ab}$. Zudem handelt es sich hierbei nicht nur um einen Abkochungsprozess. Die Herstellung des Tranks ist ein grosses heiliges Fest für alle Beteiligten «feitio» genannt - und ein eigenes Ritual zur Herstellung des heiligen Tranks «Daime». Dazu wird auch gesungen und musiziert, und die am Herstellungsprozess Beteiligten trinken während des gesamten Prozesses immer wieder schluckweise von der entstehenden Medizin. Dadurch erhalten sie die direkte Information von den Pflanzen selbst, wie lange und wie oft der Abkochungsprozess statt-
Abb. 6. Abgiessen der gekochten Flüssigkeit.
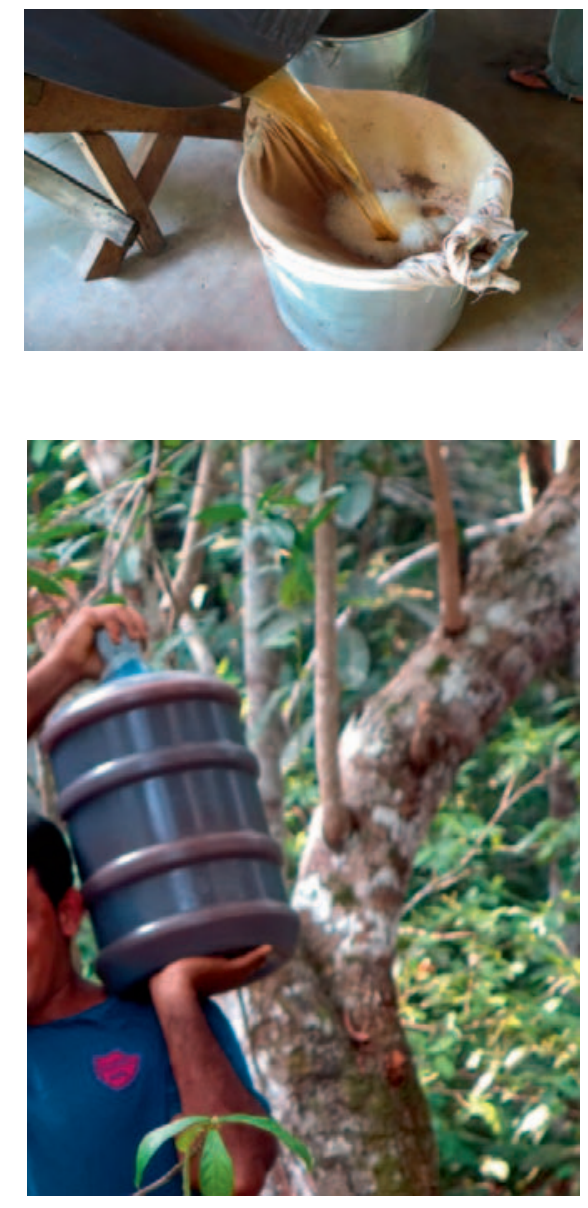

Abb. 7. Fertiges

Produkt Ayahuasca.

finden soll. Sie handeln intuitiv und deshalb jedes Mal individuell und doch nach gewissen Regeln, die nahezu dasselbe Ergebnis erzielen. Das Ritual dauert bei der kirchlichen Bewegung Santo Daime in der Regel 3 Tage und 2 Nächte, in denen in Schichten durchgekocht wird, um ein dem Geist der Medizin gerecht werdendes Produkt zu erhalten. Das Endprodukt wird einigermassen abgekühlt in Kunststoffgallonen und -flaschen abgefüllt (Abb. 7) und in einem dunklen Raum gelagert.

Was zu den Mengenverhältnissen aus den Interviews einerseits mit Vertretern der Santo-Daime-Bewegung und anderseits mit den indigenen Katukinas noch zu entnehmen war, ist, dass die indigenen Kulturen einen höheren Anteil an der Caapi-Liane verwenden, während die kirchlichen Bewegungen mehr Chacruna-Blätter hinzufügen als die indigenen Stämme. Die genauen Mengenverhältnisse konnten nicht in Erfahrung gebracht werden. Diesbezüglich hat jeder indigene Stamm bzw. jede Kirche seine/ihre eigenen Rezepte, die auch sehr individuell ausfallen. Jedes Volk, jeder Schamane, jede kirchliche Gruppe arbeitet mit den ihnen traditionell überlieferten Rezepten und Auswahl an Zutaten. Je nach gewünschter Wirkung (psychoaktiv, stimulierend, heilsam, beruhigend, reinigend usw.) werden neben der beschriebenen, meist gebräuchlichen Variante auch andere Pflanzen als Zusätze verwendet. 


\section{Anwendungen von Ayahuasca}

Ayahuasca wird seit jeher von Schamanen und Medizinmännern/Heilern indigener Völker Südamerikas, dem ursprünglichen Verbreitungsgebiet der beiden wichtigsten Pflanzen, für Heilrituale und schamanische Erfahrungen eingesetzt.

Anderseits haben sich im Laufe der Zeit sogenannte synkretistische Religionsgemeinschaften in Südamerika gebildet, die Ayahuasca als Sakrament und als Heilmittel einsetzen. Unter Synkretismus versteht man «die Synthese religiöser Ideen oder Philosophien zu einem neuen System oder Weltbild. Voraussetzung dafür ist, dass diese Ideen oder Philosophien sich zuvor als inhaltlich voneinander unterschieden abgegrenzt haben und dass sie als religiös-philosophische Teilaspekte auf einen Absolutheitsanspruch verzichten. Synkretismus nimmt vielmehr die Aspekte unterschiedlicher Religionen mehr oder weniger bewusst auf und formt sie zu etwas Neuem» [18].

\section{Schamanen - Medizinmänner - Heiler}

In der Literatur wurden bereits mehrere Versuche der Einteilung und Abgrenzung der verschiedenen Arten von Heilern im südamerikanischen Bereich gemacht $[4,7,8$, $12,19,20]$. Allen gemeinsam ist jedoch, dass die Beziehung zwischen Mensch und Natur im Mittelpunkt steht. Die Heiler wissen, dass sie in der Natur nach Weisheit suchen müssen und von der Natur lernen können. Das Wissen kommt aus diesem Verständnis von Wasser, Erde und Luft (oder Raum).

Die gebräuchlichste Bezeichnung für die südamerikanischen Heiler lautet «curandero», abgeleitet aus dem Wort curar $=$ heilen und curioso $=$ neugierig [7]. Wissensdrang ist Grundvoraussetzung, um Heiler zu werden. Dazu gehört auch der Erwerb eines Repertoires von überlieferten Riten und ethnobotanischem Wissen. Curanderos sind Arzneipflanzenkundige und Spezialisten auf dem Gebiet der Hausmittel. Je nach Ansicht der Bevölkerung können sie Krankheiten behandeln, nicht aber heilen, da sie nicht die Fähigkeiten von Schamanen besitzen, die in andere Dimensionen reisen, um nach Ursachen und Behandlungsmöglichkeiten der Krankheit zu fragen und zu suchen [4].

Schamanen verfügen über ein besonderes Wissen, was das Heilen von Krankheiten betrifft. Sie sind Vermittler zwischen der materiellen und der spirituellen Welt. Oft sind spezielle Formen des Lernens, Erfahrens und Erleidens von Krankheiten am eigenen Leib notwendig, um diese Fähigkeiten zu erlangen. Dieser Weg muss oft über Jahre erfahren werden, bevor eine Person die Initiation zum Schamanen erhält [4]. Unter den Schamanen gibt es wiederum verschiedene Klassifikationen, je nachdem mit welchen Mitteln sie arbeiten, z.B. mit psychedelischen Substanzen, mit Bäumen, mit Gerüchen oder mit Tabak [19]. Jene, die mit Ayahuasca arbeiten, werden «ayahuasceros» genannt.

In den Amazonasregionen hat sich neben vielen regionalen indigenen Bezeichnungen für Heiler der Name «payé» - eine Bezeichnung aus der indianischen TupíSprache - eingebürgert. Diese Bezeichnung leitet sich aus der Vorstellung ab, dass ein Schamane sich in einen Jaguar verwandeln kann [7].

\section{Stellenwert und Funktion}

Um die vielfältigen Anwendungen von Ayahuasca durch die Schamanen zu verstehen, ist es notwendig, das indigene, kulturbedingte Verständnis von Pflanzen, Natur und Krankheit zu erfassen. Indigene Völker haben einen sehr starken Bezug zur Natur und damit auch zu den Pflanzen. Einerseits dienen sie als körperliche Nahrung und anderseits als seelische Nahrung. Sie gelten als heilig, weil sie den Kontakt zur Anderswelt, zur unsichtbaren Welt, zur wahren Wirklichkeit, zu Göttern oder Geistern und Dämonen ermöglichen. In jeder dieser Pflanzen leben Pflanzengeister, Pflanzengötter oder Devas, mit denen man sich verbinden kann und die als Lehrer, Mütter oder Botschafter anderer Wirklichkeiten geschätzt werden. Diese heiligen Pflanzen haben Heilkraft und können Kranke von ihren Leiden befreien, schädliche Krankengeister vertreiben und auch gesunden Menschen spirituelles Wachstum bringen. Sie werden daher auch verehrt, es werden Weihrauch- und Kerzenopfer gebracht, und die Menschen begegnen ihnen ehrfurchtsvoll und mit grosser Dankbarkeit $[4,6]$.

Den indigenen Kulturen ist im Allgemeinen die Vorstellung von Krankheiten, die organisch oder körperlich bedingt sind, fremd. Für sie liegt die Ursache von Krankheiten, Schicksalsschlägen, Bedrohungen usw. in Eingriffen aus der Geisterwelt. Deshalb gehört die Einnahme von bewusstseinsverändernden Pflanzen, die ihnen helfen, mit Göttern und Ahnen in Kontakt zu treten, zu den wichtigsten Mitteln der Heilung.

Ayahuasca - «Ranke der Seele»/«Geisterliane» - weist in ihrer begrifflichen Bedeutung auf die Befreiung der Seele hin. Der Schamane reist dabei durch die Anderswelt und trifft auf seine Vorfahren oder andere Geistwesen, mit denen er in Kontakt treten kann, denen er Fragen stellen kann und von denen er Antworten bekommt. Mithilfe des Tranks ist es ihm möglich, Krankheiten zu erkennen und zu heilen, drohende Gefahren abzuwenden und zukünftige Ereignisse zu prophezeien [3, 4].

Zur Heilung einer Krankheit müssen nach deren Auffassung immer Körper und Geist behandelt werden. Ayahuasca wirkt einerseits stark auf das Bewusstsein und anderseits auf den Körper, indem der Trank, sofern dies zur 
Heilung notwendig ist, auch Erbrechen und/oder Durchfall auslösen kann. Diese Wirkungen stehen im zentralen Mittelpunkt der schamanistischen Auffassung - Reinigung und Befreiung [4].

Einige Schamanen bereiten sich auf die Rituale nach ganz bestimmten Vorschriften vor, wie sexuelle Enthaltsamkeit, Diät oder Fasten und Waschungen $[4,6]$.

Bei einem schamanistischen Heilungsritual trinken immer sowohl der Schamane als auch der Heilungssuchende die Medizin. Somit sind beide an die andere Wirklichkeit, an die Götter, an das grosse Ganze angebunden. Der Schamane erkennt die Ursachen für die Krankheit beim Heilungssuchenden und unterstützt ihn bei den einzelnen Heilungsschritten, die der Heilungssuchende jedoch selbst beschreiten muss. Ohne Bereitschaft und Willen des Heilungssuchenden zur Änderung/Veränderung kann der Schamane nicht helfend wirken.

Einen wesentlichen Bestandteil bei der Durchführung des Heilungsrituals bilden die sogenannten «icaros», spezielle Lieder (Heilgesänge), die oft mit begleitenden Instrumenten wie Trommeln, Rasseln, Flöten, Pfeifen und Gitarren gesungen werden, um die Visionen zu lenken, zu verstärken, zu heilen, Geister zu rufen oder den Schamanen und den Heilungssuchenden zu schützen [7].

\section{Anwendung im kirchlich-religiösen Bereich}

Ayahuasca kommt auch im kirchlich-religiösen Bereich zum Einsatz, und zwar in drei kirchlichen Bewegungen, die in Brasilien entstanden und mittlerweile auch schon in einigen europäischen Staaten Verbreitung gefunden haben.

Die drei brasilianischen Ayahuasca-Religionen Santo Daime, Barquinha und UDV sind in einer ähnlichen Situation und Umgebung sowie unter ähnlichen Umständen und Voraussetzungen entstanden, haben sich aber - basierend auf einem unterschiedlichen emischen Diskurs - aufgrund unterschiedlicher Missionen verschieden entwickelt. Die Übereinstimmungen liegen in der Entstehung dieser Religionen in einem gemeinsamen oder ähnlichen ökonomischen, sozialen und kulturellen Kontext in der Region Acres und in Epochen des Niedergangs des ersten (1850-1920) und zweiten Kautschuk-Rauschs (während des Zweiten Weltkriegs ab 1943) [12]. Diese Religionen entstanden gleichsam aufgrund von Offenbarungserlebnissen aus dem Nordosten Brasiliens eingewanderter Migranten. Die Offenbarungserlebnisse, das Empfangen der Mission, erfuhren alle drei Gründer dieser Religionen in Ayahuasca-Ritualen. Die zentrale Gemeinsamkeit dieser drei Religionen ist, neben ihrem ähnlichen Ursprung in ähnlicher Situation, der kollektive und sakramentale Gebrauch Ayahuascas in allerdings sehr unterschiedlichen Ritualen und - verkürzt gesagt - als Ziel,
Abb. 8. Padrinho Alfredo, Führer der Santo-Daime-Gruppe CEFLURIS.

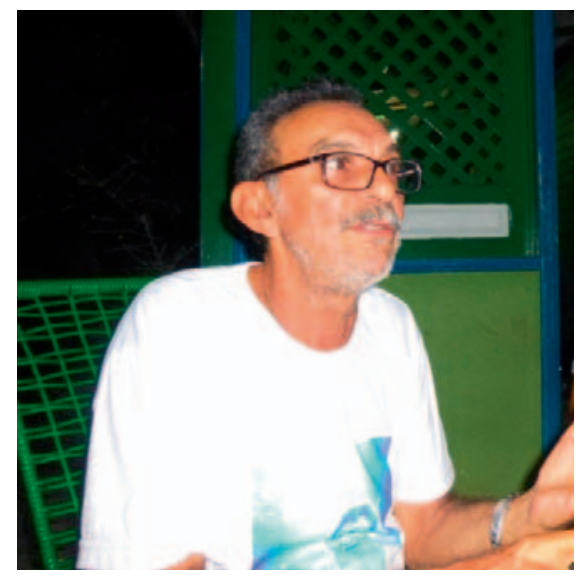

Wirkung oder Folge dieser Rituale eine «Re-Organisation des eigenen Lebens» der Anhänger dieser drei Religionen [12].

Aus einem persönlichen Interview mit dem derzeitigen Führer der Santo-Daime-Gruppe CEFLURIS (Centro Ecléctico de Fluente Luz Universal Raimundo Irineu Serra - «Eklektisches Zentrum des Fliessenden Universellen Lichtes Raimundo Irineu Serra»), Alfredo Gregório de Melo (Padrinho Alfredo) über die Doktrin der Glaubensgemeinschaft und deren Entstehung wie auch ihrer Verbreitungsbestrebungen konnte Folgendes entnommen werden:

In den 1930er-Jahren gründete Raimundo Irineu Serra, genannt Mestre Irineu, die Lehre des Santo Daime in Rio Branco (Bundesstaat Acre). Es handelte sich um einen in Brasilien eingewanderten Afroamerikaner, der durch Kontakt mit der indigenen Bevölkerung mit Ayahuasca in Berührung gekommen war und aufgrund eines Offenbarungserlebnisses unter Einwirkung dieses Tranks einen neuen christlichen Weg beschritten hatte. Sein Nachfolger war Sebastião Mota de Melo, genannt Padrinho Sebastião, dessen Initiation ebenfalls durch das Daime (= Ayahuasca-Trank), das er von Mestre Irineu erhalten hatte, geschah. Der Trank wurde von Mester Irineu in "Daime» umbenannt, was "gebt mir» - dai = geben, $\mathrm{me}=\mathrm{mir}-$ bedeutet. Im täglichen Sprachgebrauch der Kirchengemeinschaft wird auch von «Tee» oder «Wein» gesprochen.

Aus der Ursprungsbewegung Santo Daime spalteten sich im Laufe der 1970er- und 1980er-Jahre hauptsächlich drei Untergruppen ab: CICLU, CECLU und CEFLURIS. CEFLURIS ist die derzeit bekannteste Santo-DaimeGruppe, deren Gründer Padrinho Sebastião war. Sein Sohn, Alfredo Gregório de Melo (Padrinho Alfredo, Abb. 8), leitet wie bereits erwähnt diese heute weltweite Bewegung.

Mittlerweile umfasst diese Gruppierung laut Aussage von Padrinho Alfredo weltweit ca. 45000 offizielle Mitglieder, unter anderem in den USA sowie in Japan, Aus- 


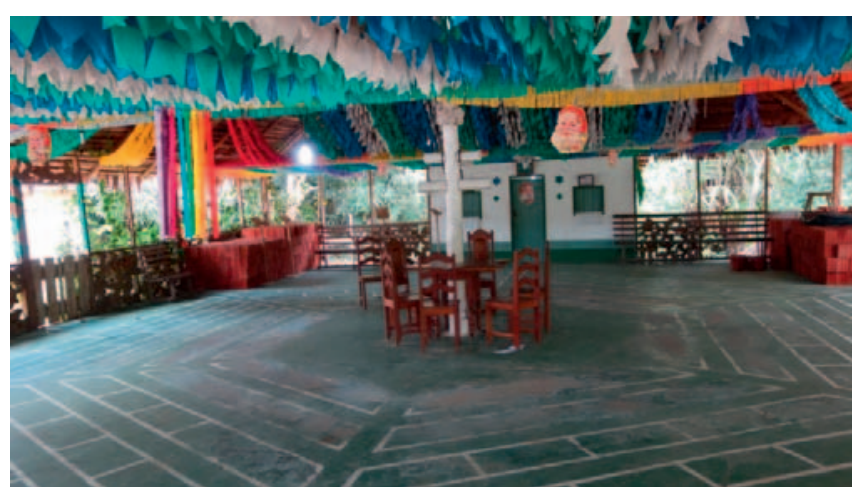

Abb. 9. Santo-Daime-Kirche mit sechszackigem Tisch und Bodenmarkierungen für Aufstellung bei Zeremonien.

tralien und Europa. Ebenso berichtete er, dass in jedem einzelnen Land/Staat der Expansion die Bemühungen um die Legalisierung des Daime-Getränks zum Einsatz als Sakrament im religiösen Gebrauch laufen. In Brasilien selbst wie auch in Oregon (USA) und in den Niederlanden konnte dies erreicht werden.

\section{Stellenwert und Funktion, speziell bei Santo Daime}

Die Santo-Daime-Mitglieder leben nach der Lehre von Mestre Irineu, genannt «die Doktrin». Gemäss dieser trinkt man Daime anlässlich von Zeremonien und Festen des Kirchenkalenders. Hauptsächlich vier Arten von Santo-Daime-Ritualen werden innerhalb der Gemeinschaft praktiziert: die festas officiais (offizielle Feiertage), die trabalhos de concentracão (Konzentrationsrituale), die missas (Messen) und die trabalhos de cura (Heilrituale) [12].

Balzer [12] beschreibt die einzelnen Rituale folgendermassen: «Die offiziellen Feste (festas officiais) folgen dem christlichen Kalender. Zusätzlich wurde der 15. Dezember, der Geburtstag des Gründers Raimundo Irineu Serra, aufgenommen. Mittlerweile wurden auch die Geburtstage der Nachfolger aufgenommen und noch einige andere, sodass sich die Anzahl auf 19 sogenannte 〈hinarios> beläuft. Die festas beginnen heute einheitlich um 18 Uhr und dauern 6-12 Stunden. Zu Beginn des Rituals werden von den Teilnehmenden gemeinsam 3 Vater Unser, 3 Ave Maria und 1 Gebenedeit seist du Maria sowie einige Doktrinspezifische Anrufungen gebetet. Danach wird das Sakrament der 〈heilige Daime` verteilt. Der eigentliche RitualInhalt besteht im gemeinsamen Singen der Hymnen und im gemeinsamen Tanz, wobei einzelne Mitglieder verschiedene Musikinstrumente (Rassel, Gitarre, Violine, Flöte) spielen. Das Ritual endet mit dem Rezitieren christlicher Gebete und Anrufungen an bestimmte, christliche Heilige.»

Diese Handlungen finden in der Kirche in einer ganz bestimmten vorgegebenen Aufstellung um einen sechszackigen Sternentisch (Abb. 9) und unter genau definier-

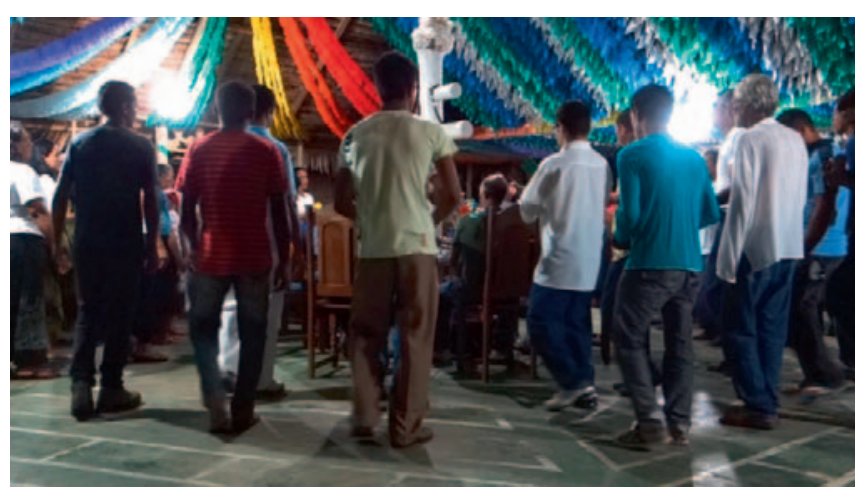

Abb. 10. Kirchliche Santo-Daime-Zeremonie mit Tanz.

ten Schrittbewegungen der Mitglieder statt (jeweils drei Schritte nach links und drei Schritte nach rechts, wie ich aus meiner teilnehmenden Beobachtung an einer "missa» der Santo Daime mitverfolgen konnte), wobei Männer und Frauen voneinander und auch innerhalb dieser nochmals nach Altersgruppen getrennt sind (Abb. 10).

Die Konzentrationsrituale finden am 15. und 30. jeden Monats statt und dienen der spirituellen Entwicklung der Teilnehmenden. Die Rituale beginnen um 19 Uhr und dauern 2-4 h, wobei das Sakrament nur einmalig verteilt wird. Nach dem Verteilen des Sakraments und dem Sprechen der Gebete herrscht Stille, um die Konzentration (Meditation) zu ermöglichen. Die Teilnehmenden sitzen auf einfachen Holzbänken. Insgesamt gibt es 24 Konzentrationsrituale pro Jahr.

Neben diesen «offiziellen Terminen» finden jede Menge nicht offizielle Feiern in Form von Messen (diese dauern in der Regel 2-3 h) und Heilarbeit statt, sodass die Mitglieder im Jahr auf insgesamt ca. 80-90 Rituale kommen [21].

Die einzelnen Rituale werden von den Mitgliedern als spirituelle Arbeit an sich selbst gesehen. Die Doktrin ruft auch dazu auf, alle spirituellen Lektionen im Alltag umzusetzen [13].

Nach Ansicht der Mitglieder wirkt der Trank auf den Körper reinigend und entgiftend, führt $\mathrm{zu}$ innerem Gleichgewicht, ist für die Diagnosestellung durch Autoanalyse wichtig und reinigt den Geist [4].

Padrinho Alfredo charakterisiert Daime folgendermassen [22]:

«Santo Daime, it is very powerful and can open channels of wisdom for all of us - wisdom of the forest, wisdom of the planet, wisdom of our own salvation. We all can have a knowledge that brings us peace, spiritual peace, family peace, so we can recognize each other as a family and work for this good.»

Im Daime-Kult gilt die Caapi-Liane als Verkörperung von Jesus, die Psychotria-Blätter als Maria [6]. Dies bestätigte mir Padrinho Alfredo im Interview. So stellt der Höhepunkt des Rituals das miração dar, die Offenbarung, die 
sich in einer Heiligenerscheinung zeigen kann oder zur «Einsicht» führt [14]:

«The miração, which can be translated as «the act of seeing», is an experience often accompanying the drinking of daime. The miração brings along transformative insights that can change an individual's self-understanding and his/her relatedness to the rest of the world.»

\section{Vergleich der beiden Anwendungsbereiche}

Vergleicht man nun die unterschiedlichen Anwendungen durch Schamanen und durch Kirchen, so unterscheiden sich diese hauptsächlich in der Form der Durchführung. Also sind lediglich die Rituale, in denen Ayahuasca als Medizin oder als Sakrament eingebettet ist, von unterschiedlicher Art. Ritual wird definiert als «eine nach vorgegebenen Regeln ablaufende, meist formelle und oft feierlich-festliche Handlung mit hohem Symbolgehalt. Sie wird häufig von bestimmten Wortformeln und festgelegten Gesten begleitet und kann religiöser oder weltlicher Art sein (z.B. Gottesdienst, Begrüssung, Hochzeit, Begräbnis, Aufnahmefeier usw.)» [23].

Sowohl bei den Schamanen als auch bei den Kirchen orientieren sich die Abläufe beim Einsatz von Ayahuasca nach zwar unterschiedlichen, aber vorgegebenen, oft in langen Traditionen überlieferten Regeln.

Dabei spielen "Set und Setting» eine zentrale Rolle. Hierbei handelt es sich um eine Theorie, die von Timothy Leary, einem amerikanischen Psychologen, der wissenschaftliche Experimente mit psychedelischen Substanzen (Lysergsäurediethylamid (LSD), Psilocybin) durchführte, entwickelt wurde $[4,6]$. Sie besagt, dass für bewusstseinsverändernde Erfahrungen durch psychoaktive Stoffe drei Faktoren wichtig sind: die Dosis, das Set und das Setting. Set ist die innere Einstellung, die Intention des Menschen sowie seine Erwartung und seine Konstitution. Das Setting umfasst die Umgebung, den Ort, die Zeit und den Raum, in dem das Geschehen stattfindet. Alle diese Komponenten zusammen bewirken die Veränderung im Denken, im Fühlen, in den Emotionen, in der Wahrnehmung, im Köpererleben und im Selbsterleben $[4,6]$.

Möglicherweise sind diese Faktoren weder den Schamanen noch den Heilungssuchenden noch den Führern der kirchlichen Bewegungen und deren Mitgliedern bewusst. Dennoch arbeiten sie alle sehr intensiv damit, aus welchen Gründen auch immer: aus Überlieferung, aus Erfahrung, aus rituell vorgegebenen Regeln.

Weitere Unterschiede bestehen in der Art der Zubereitung des Tranks und den verschiedenartigen Zusätzen zur Caapi-Liane je nach gewünschter Wirkung.

Die Ziele beider Gruppen - der Schamanen und der Kirchen - sind schlussendlich die gleichen. Beide streben sowohl das seelisch-geistige als auch das körperliche Wohlbefinden (Heil) und das spirituelle Wachstum an.
Ayahuasca ist dabei ein «Hilfsmittel», das einerseits als Kommunikationsmittel zur Kontaktaufnahme mit den Ahnen, den Geistern, den Göttern, der Natur und mit anderen Welten dient und das anderseits Einsichten und Wissen vermittelt. Ziel ist es, die Einsichten im täglichen Leben anzuwenden bzw. Änderungen im Leben herbeizuführen, die zu einem liebevollen und harmonischen Umgang grundsätzlich mit allen Lebewesen, vor allem aber in der Familie der Stammes-/Glaubensgemeinschaft, anleiten.

Auf körperlicher Ebene dient es der inneren Reinigung und Heilung, die unterstützt durch die seelisch-geistige Weiterentwicklung umso rascher voranschreitet. Diese Prozesse gehen Hand in Hand.

Eine weitere Gemeinsamkeit der beiden beschriebenen Gruppen, trotz unterschiedlicher Wege, Anwendungen und Zugänge, liegt im Ursprung, in der gemeinsamen Wurzel: nämlich, dass sie Kontakt zu Ayahuasca über ein oder mehrere indigene Völker erhalten haben.

\section{Ausblick}

Von den Wissenschaftern Charles Grob, Dennis McKenna und James Callaway wurde in den 1990er-Jahren ein interdisziplinäres Forschungsprojekt unter dem Zusammenschluss verschiedener Universitäten aus Kalifornien, Finnland und Brasilien, genannt «Hoasca project», durchgeführt, in dem die medizinischen, pharmakologischen, psychiatrischen und gesundheitlichen Auswirkungen regelmässigen Ayahuasca-Gebrauchs an den Mitgliedern der UDV-Bewegung in Brasilien untersucht wurden. Die Ergebnisse der Pilotstudie wurden in zahlreichen "peer-reviewed papers» veröffentlicht und in einer ausführlichen Besprechung von McKenna [24] zusammengefasst.

Die Hauptergebnisse brachten zutage, dass langjährige UDV-Mitglieder ihr Leben und ihr Verhalten in positiver und profunder Weise geändert hatten. Der laufende Gebrauch von Ayahuasca im rituellen Kontext und in der sozial unterstützenden Umgebung der UDV wurde als sicher eingestuft, hat keine Langzeitwirkungen wie Giftigkeit und zeitigt dauerhaften positiven Einfluss auf die physische und mentale Gesundheit - sie wird von McKenna als eine therapeutisch wertvolle Medizin bezeichnet [9]. Verhaltensänderungen der Probanden wurden folgendermassen beschrieben: ruhiger, gemässigter, ordentlicher, beharrlicher, loyaler, überlegter. Daneben sind auch Änderungen in der Gemütslage beobachtet worden: Die Probanden waren zuversichtlich, entspannt, optimistisch, sorgenfrei, hemmungsloser und kontaktfreudig. Ausserdem zeigten die Langzeitanwender von Ayahuasca ein höheres Mass an Konzentration und eine Verbesserung des Kurzzeitgedächtnisses. 
Alkohol- und Zigarettenabhängige sowie gelegentlich drogenkonsumierende Probanden berichteten von einer radikalen Umstrukturierung ihrer persönlichen Lebensführung und ihres Wertesystems, sodass sie Alkohol-, Zigaretten- und Drogenkonsum nicht mehr weiterverfolgten.

So wurde Ayahuasca im Laufe der Zeit immer mehr zur Heilung von Suchterkrankungen eingesetzt.

Am oberen Amazonas in Peru gründete z.B. Dr. Jaques Mabit, ein französischer Schulmediziner, im Jahr 1992 das Heilzentrum von TAKIWASI («Singen der Bäume») zur Behandlung von Suchtkrankheiten und zur Erforschung traditioneller Medizin. Generell bestehen die Aufgaben Dr. Mabits und TAKIWASIs darin, die menschlichen und natürlichen Ressourcen der traditionellen Medizin einer Neubewertung zuzuführen und eine tatsächliche therapeutische Alternative zur Behandlung von Suchterkrankungen zu entwickeln, die nicht nur wirksam und kostengünstig ist, sondern auch den Bedürfnissen von Patienten angepasst werden kann, die einen unterschiedlichen kulturellen und sozialen Hintergrund aufweisen.

Die Erfolgsrate von Dr. Mabit und seinem Rehabilitationszentrum ist im Vergleich zur konventionellen schulmedizinischen Therapie von Drogenabhängigen sehr hoch. Während die moderne Schulmedizin mit ihren Methoden eine Erfolgsrate von lediglich 5\% bei der dauerhaften Entwöhnung von Suchtkrankheiten aufweist, kann Dr. Mabit mit der von ihm angewandten schamanisch orientierten Methode auf eine solche von beeindruckenden 33\% verweisen. Ein Drittel aller Patienten werden in TAKIWASI tatsächlich gänzlich von ihrer Sucht befreit, ohne dass es jemals zu Rückfällen kommt.

Im Vordergrund seiner Therapie steht die Verabreichung von Ayahuasca, das seinen drogenabhängigen $\mathrm{Pa}$ tienten die wahren Ursachen ihrer Sucht bewusst macht. Die Verabreichung dieser entheogenen Pflanze zu therapeutischen Zwecken wird stets von schamanischen Ritualen begleitet, welche die Visionen und inneren Erlebnisinhalte der Patienten in eine für deren Therapie taugliche Richtung steuern und ihnen letztendlich die tiefer liegenden Ursachen der Sucht enthüllen. Sobald die individuelle(n) Ursache(n) der Suchterkrankung abgeklärt ist (sind), setzt die Therapie zu deren Beseitigung ein. Dabei werden sowohl Konzepte der modernen Psychotherapie als auch der schamanischen Medizin sowie bestimmte Kräuter und Parfums eingesetzt, die der Reinigung, Entgiftung und Entschlackung des Körpers des Patienten dienen bzw. dessen gesamtes psycho-physisches System harmonisieren und wieder in Balance bringen $[25,26]$.

Neuere Studien, wie z.B. die von José Carlos Bouso [27], Doktor der Pharmakologie und Psychologe, über Mitglieder zweier brasilianischer Kirchen (CEFLURIS und Barquinha) kommen zu ähnlichen Ergebnissen wie im «Ho-

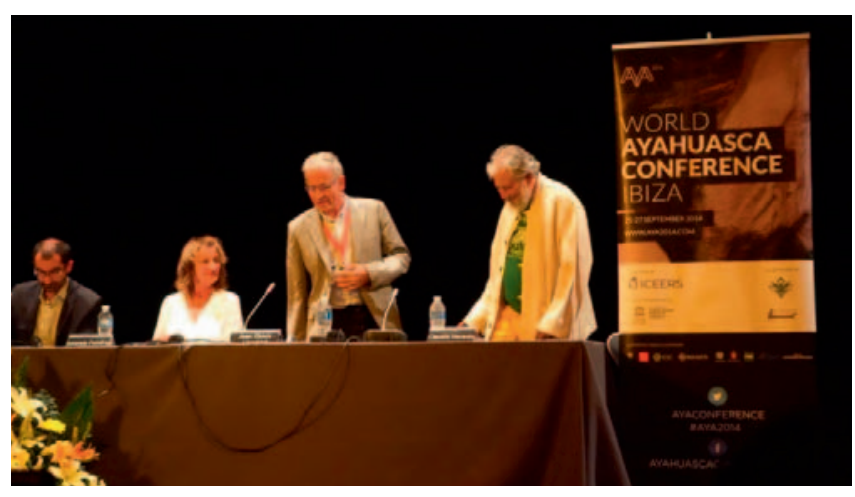

Abb. 11. World Ayahuasca Conference 2014, Ibiza.

asca Project», d.h., dass Ayahuasca einen positiven Einfluss auf verschiedensten Ebenen für den Menschen hat [27]:

«The assessment of the impact of long-term ayahuasca use on mental health from various perspectives (personality, psychopathology, neuropsychology, life attitudes and psychosocial well-being) did not find evidence of pathological alterations in any of the spheres studied. Although ayahuascausing subjects differed in some personality traits, differences did not fit with a pathological profile. Furthermore, ayahuasca users showed a lower presence of psychopathological symptoms compared to controls. They performed better in neuropsychological tests, scored higher in spirituality and showed better psychosocial adaptation as reflected by some attitudinal traits such as Purpose in Life and Subjective Well-Being. Overall differences with the control group were still observable at follow-up one year later.»

Weiterhin ergab die Studie, dass es zu keinerlei Abhängigkeit bei der Langzeitanwendung von Ayahuasca kommt [27]:

«We assessed addiction severity in two samples of long-term members of the ayahuasca religions and we did not find participants to be saddicted s to the tea. Nor did we observe the deleterious psychosocial effects commonly associated with drugs of abuse.»

Ebenso kommen Callaway et al. [28] und Gable [29] zu dem gleichen Ergebnis, dass Ayahuasca nicht abhängig macht und nicht gesundheitsschädlich ist.

Weltweit ist ein zunehmendes Interesse an der Erforschung und Verbreitung von Ayahuasca zu erkennen. Dies zeigte auch die World Ayahuasca Conference auf Ibiza (25.-27.09.2014), die von ICEERS (International Center for Ethnobotanical Education, Research \& Service) veranstaltet wurde und unter der Schirmherrschaft der UNESCO stand (Abb. 11).

ICEERS ist eine philanthrope, steuerbefreite Non-Profit-Organisation, die sich folgenden Aufgaben widmet: 1) der Integration von Ayahuasca, Iboga und anderen traditionellen Pflanzen als therapeutisches Werkzeug in der modernen Gesellschaft und 2) der Erhaltung von indigenen Kulturen, die diese Pflanzenarten seit der Antike nutzen, deren Lebensräumen und deren botanischen Ressourcen.

Hier versammelten sich Wissenschafter, Ärzte, Forschende, Rechtsanwälte, Vertreter von Drogenbehörden 
und Vertreter indigener Völker aus 50 Nationen, um einerseits Ayahuasca und seine Wirkungen näherzubringen und anderseits den Austausch über dieses weitläufige Thema in allen Disziplinen zu pflegen. Unter anderem fanden sich dort auch die zuvor erwähnten Dr. Dennis McKenna und José Carlos Bouso. Die Konferenz hatte etwa 500 Besucher.

Die Themen erstreckten sich vom indigenen, religiösen und klinischen Gebrauch von Ayahuasca bis zur persönlichen Entwicklung durch die Anwendung des Tranks. Auch Fragen der ethnobotanischen und philosophischen Sichtweise bis hin zur wissenschaftlichen Forschung waren Thema der Konferenz. Ausserdem wurde die rechtliche Seite im Sinne der Legalisierung und Verbreitung von Ayahuasca und der Menschenrechte beleuchtet. Weitere Themenkreise bildeten die Globalisierung und Nachhaltigkeit, der Schutz der Pflanzen und die Rolle der indigenen Frau in der Welt des Ayahuasca. Abgerundet wurde das Programm durch Erfahrungsberichte, Präsentationen derzeit laufender Forschungen, Bücher und Filme.

Im Teil «Klinische Forschung» wurden Studien und Erkenntnisse über die Wirkung von Ayahuasca insbesondere auf das menschliche Gehirn präsentiert. Daraus werden zwei interessante wissenschaftliche Ansätze exemplarisch herausgegriffen: Nach einhelliger Ansicht der bisherigen Forschung verstärkt Ayahuasca den Blutfluss im Gehirn, sodass einige Teile des Gehirns mehr versorgt werden als andere. Dr. Jordi Riba, Pharmakologe und Professor an der Autonomen Universität Barcelona/Spanien und Forschungsmitarbeiter im Sant Pau Hospital Barcelona (Vortrag am 25.9.2014), war der Ansicht, dass dies zur Aktivität gewisser Teile im Gehirn führt, die ohne Einflussnahme von Ayahuasca nicht tätig werden. Dr. Riba geht davon aus, dass der Mensch in der Aufnahme von Informationen nicht neutral ist. Der Mensch interpretiert immer. Ayahuasca setzt nun in einem Teil des Gehirns an, der zwischen dem emotionsauslösenden Bereich und dem Erkenntnisbereich liegt. Es modifiziert die Erwartungen und fördert den inneren Dialog, sodass ein neuer Blick auf die Realität ausgelöst wird. Er spricht davon, dass Selbsttranszendenz und Spiritualität zunehmen und das «Ego» zurückgedrängt wird - basierend auf der Studie von Riba et al. [15].

Einen weiteren interessanten Ansatz lieferte Dr. Draulio Barros de Araujo, Doktor der medizinischen Physik: Er geht davon aus, dass Ayahuasca auf das DMN (default mode network) im Gehirn wirkt. Das DMN ist ein beständiges Netzwerk im Gehirn (Teil des Gehirns), das an Aktivität zunimmt, wenn sich der Mensch in Ruhe befindet. Es werden in diesem Zustand von dort vermehrte Signale ausgesendet, die zu Tagträumen führen, zum Wandern von Gedanken. Er konnte beobachten, dass Ayahuasca auf das DMN in der Weise wirkt, dass es zu einer Reduktion der Signalaussendung kommt und damit $\mathrm{zu}$ einer
Reduktion der Gedankenwanderung. Dieses Phänomen konnte auch bei Menschen in Meditation festgestellt werden.

Diese Wirkung von Ayahuasca kann bei der Behandlung von Depressionen eine grosse Rolle spielen. Bei der Depression kommt es zur Bildung von negativen Gedanken und negativen Tagträumen, die durch die eben beschriebene Wirkung von Ayahuasca auf das DMN reduziert bzw. eingedämmt werden können.

Die Konferenz liess einen eindeutig verstärkten Trend in Richtung medizinischer Erforschung von Ayahuasca und dessen Wirkungen zum Zweck des Einsatzes bei der Behandlung von verschiedenen Krankheiten und bei der persönlichen Entwicklung erkennen - und das in den verschiedensten Staaten der Welt.

\section{Fazit: Ayahuasca: Medizin des Regenwaldes - Medizin für Europa?}

Bisher konnten durch die Anwendung von Ayahuasca keine nachteiligen Nebenwirkungen (auch nicht im Langzeitbereich) oder eine Entwicklung von Abhängigkeit und Sucht entdeckt werden. Es stellt sich daher die Frage: Kann die Medizin des Regenwalds auch eine Medizin für Europa sein?

Grundsätzlich spricht nach den vorangegangenen Ausführungen über die verschiedensten positiven Wirkungen nichts dagegen. Ein wesentlicher Aspekt, der bei der Anwendung von Ayahuasca jedoch nicht ausser Acht gelassen werden sollte, sind, wie bereits angesprochen, Set und Setting. Dieser psychoaktive Trank wurde seit jeher in einem zeremoniellen Rahmen eingenommen, in Achtung und Würdigung sowohl der Pflanzen (Natur) als auch der Menschen, die sich bewusst mit sich selbst und der Natur auseinandersetzen. Genauso bewusst sollte daher auch heute bei der Anwendung in anderen Kulturen mit dieser «Medizin» umgegangen werden. Ayahuasca soll kein schnelles Vergnügen in unserer heutigen schnelllebigen Zeit bieten; vielmehr ist das Hauptgewicht auf Heilung auf den verschiedensten Ebenen zu legen. Ein achtsamer Umgang, wie er auch bei Menschen untereinander gepflegt werden sollte, ist auch bei der Arbeit mit dieser Medizin sehr wichtig. Die entsprechende Intention des Einzelnen hierzu, der geeignete Rahmen in bestimmt definierten Abläufen und der betreuende, im Umgang mit dieser Medizin vertraute Helfer (Schamane, Heiler) sind daher essenzieller Teil der Anwendung. Diese Faktoren bieten dem Anwender Halt, geben ihm Rahmen und Stütze und erhöhen seine Bewusstheit, Aufmerksamkeit und Achtsamkeit sich und seiner Umwelt gegenüber.

Da bisher in sehr vielen Staaten von Europa Ayahuasca aufgrund der darin enthaltenen Substanz DMT nicht legal ist, konnte sich lediglich in den Niederlanden eine 
Station der Santo-Daime Bewegung-etablieren, da in diesem Land die Anwendung von Ayahuasca im Rahmen der kirchlichen Zeremonien als zulässig erklärt wurde. In Teilen Portugals ist die Anwendung zulässig. Zeremonien werden dort öffentlich im Internet angeboten. In Spanien arbeitet derzeit die Santo-Daime-Kirche an der Legalisierung. In Deutschland ist DMT als Betäubungsmittel eingestuft und der Umgang damit, ohne spezielle Erlaubnis, nicht erlaubt. In Österreich fällt DMT unter das Suchtmittelgesetz, das den Besitz, den Erwerb und die Weitergabe verbietet. Hierunter fallen nicht DMT-haltige Pflanzen. Auf einigen Internetseiten werden Ayahuasca-Zeremonien in Österreich öffentlich angeboten.

Es ist ersichtlich, dass die Bestrebungen, Ayahuasca auch im europäischen Raum zu verbreiten, vorhanden sind. Wie weit diese Entwicklung gehen kann und wird, lässt sich zum derzeitigen Zeitpunkt schwer abschätzen. Dass Ayahuasca eine Bereicherung auf verschiedenen Ebenen sein kann, bleibt unbestritten, und die zukünftigen Forschungsergebnisse wie auch die Offenheit der Menschen und der achtsame Umgang werden den weiteren Entwicklungsweg dieser speziellen Pflanzenmedizin bestimmen.

\section{Danksagung}

Mein besonderer Dank gilt allen Beteiligten, denen ich auf meiner Expedition im Februar 2014 in Acre/Brasilien begegnen durfte und die mir ihr Gedankengut wie auch die Medizin nähergebracht haben. Es war für mich eine grosse Ehre und wunderbare Erfahrung, die Pflanzen an ihrem Standort im Regenwald kennenlernen und den gesamten Herstellungsprozess der Medizin in einer «feitio» miterleben zu dürfen. Meine Achtung vor der Natur und vor den Menschen, die dort im Einklang mit der Natur leben und sich durch sie führen lassen, erfuhr eine weitere Steigerung. Ausserdem bedanke ich mich recht herzlich bei Padrinho Alfredo, dem derzeitigen Vater der Santo-Daime-CEFLURIS-Bewegung, der geduldig für das Interview zur Verfügung gestanden ist und mir tiefere Einblicke in die Welt dieser Glaubensgemeinschaft gegeben hat.

\section{Disclosure Statement}

In Bezug auf diese Arbeit bestehen keine Interessenkonflikte. Sylvia Linhart ist diplomierte Kräuterpädagogin und Heilkräutercoach und arbeitet in Wien wie auch anderen Bundesländern Österreichs. Sie hat Ausbildungen bezüglich Heilpflanzenwissen in Aflenz/Steiermark, Österreich, wie auch bezüglich Ethnobotanik am Ethnobotanischen Institut in Freisen-Grügelborn, Deutschland, absolviert. In Österreich erhielt sie das Diplom zur Kräuterpädagogin. Ausserdem hat sie das CAS «Ethnobotanik und Ethnomedizin» an der Universität Zürich, Schweiz, abgeschlossen, in dessen Rahmen diese Projektarbeit geschrieben wurde. Ihr Interessengebiet gilt dem Einfluss der Natur auf das Wohlbefinden des Menschen.

\section{Literatur}

1 Schultes RE, Raffauf RF: The Healing Forest. Medicinal and Toxic Plants of the Northwest Amazonia. Portland, Dioscorides Press, 1990, pp 48, 274-281, 395.

2 Balick MJ, Cox PA: Drogen, Kräuter und Kulturen. Pflanzen und die Geschichte des Menschen. Heidelberg, Spektrum, 1997, pp 172-177.

3 Schultes RE, Hofmann A: Pflanzen der Götter. Die magischen Kräfte der bewusstseinserweiternden Gewächse, ed 5. Aarau, AT, 2001, pp 124-139.

4 Prentner A: Bewusstseinsverändernde Pflanzen von A - Z. Wien, Springer, 2005, pp 10 11, 36-41, 68-70, 256-260.

5 Mullen A: Psychoaktive Pflanzen, Pilze und Tiere. Bestimmung, Wirkung, Verwendung, ed 3. Stuttgart, Franckh-Kosmos, 2011, pp 14-16.

6 Rätsch C: Enzyklopädie der psychoaktiven Pflanzen. Botanik, Ethnopharmakologie und Anwendungen, ed 10. Aarau, AT, 2012, pp 9-21, 86-88, 456-458, 702-715, 831-834.

7 Greifeld K (Hrsg): Medizinethnologie. Eine Einführung (Ethnologische Paperbacks). Berlin, Dietrich Reimer, 2013, pp 58-63.

8 Labate BC, Cavnar C (eds): Ayahuasca Shamanism in the Amazon and Beyond. New York, Oxford University Press, 2014, pp 3, 59-60, 64-65, 69.

9 Metzner R (ed): The Ayahuasca Experience. A Sourcebook on the Sacred Vine of Spirits. Rochester, Park Street Press, 2014, pp 1, 33 $35,57,66$.

10 Wink M, van Wyk BE, Wink C: Handbuch der giftigen und psychoaktiven Pflanzen. Stuttgart, WVG, 2008, pp 10, 63, 255-256.
11 http://de.wikipedia.org/wiki/Halluzination (Zugriff am 25.10.2014).

12 Balzer C: Wege zum Heil. Die Barquinha. Eine ethnologische Studie zu Transformation und Heilung in den Ayahuasca-Ritualen einer brasilianischen Religion. Mettingen, Brasilienkunde, 2013, pp 40-48, 52-53, 68, 206.

13 Polari de Alverga A: Der Prophet aus dem Regenwald. Begegnung mit heiligen Pflanzen. Burgrain, KOHA, 2007, p 9.

14 Schmidt TK: Morality as Practice. The Santo Daime, an Eco-Religious Movement in the Amazonian Rainforest. Uppsala, Acta Universitatis Upsaliensis, 2007, pp 101, 145.

15 Riba J, Romero S, Grasa E, Mena E, Carrió I, Barbanoj MJ: Increased frontal and paralimbic activation following ayahuasca, the panAmazonian ebriant. Psychopharmacology (Berl) 2006;186:93-98

16 Müller-Ebeling C, Rätsch C, Storl WD: Hexenmedizin. Die Wiederentdeckung einer verbotenen Heilkunst. Schamanische Traditionen in Europa, ed 8. Aarau, AT, 2011, p 241.

17 McKenna DJ: Clinical investigations of the therapeutic potential of ayahuasca: rationale and regulatory challenges. Pharmacol Ther 2004; 102:111-129.

18 http://de.wikipedia.org/wiki/Synkretismus (Zugriff am 12.10.2014).

19 Von Lüpke G: Altes Wissen für eine neue Zeit. Gespräche mit Heilern und Schamanen des 21. Jahrhunderts. München, Kösel, 2012, pp 155-168.
20 Helman CG: Culture, Health and Illness, ed 5. Boca Raton, FL, CRC Press (Taylor \& Francis Group), 2007, pp 81-87, 221-223.

21 Dawson A: Santo Daime. A New World Religion. London, Bloomsbury, 2013, p 50.

22 Persönliches Interview mit Padrinho Alfredo in Rodriguez Alves, Bundesstaat Acre, Brasilien, vom 08.02.2014.

23 http://de.wikipedia.org/wiki/Ritual (Zugriff am 14.10.2014).

24 McKenna DJ, Grob CS, Callaway JC: The scientific investigation of ayahuasca: a review of past and current research. Heffter Rev Psychedelic Res 1998;1:65-77.

25 http://balishaman.com/Solche_Neoschamanen_ braucht_die_Welt-Teil04.htm (Zugriff am 12.10.2014).

26 http://www.takiwasi.com/indexen.html (Zugriff am 15.10.2014).

27 Bouso JC, González D, Fondevila S, Cutchet M, Fernández X, Ribeiro Barbosa PC, Alcázar-Córcoles MÁ, Araújo WS, Barbanoj MJ, Fábregas JM, Riba J: Personality, psychopathology, life attitudes and neuropsychological performance among ritual users of Ayahuasca: a longitudinal study. PLoS One 2012; 7:e42421.

28 Callaway JC, McKenna DJ, Grob CS, Brito GS, Raymon LP, Poland RE, Andrade EN, Mash DC: Pharmacokinetics of Hoasca alkaloids in healthy humans. J Ethnopharmacol 1999;65:243-256.

29 Gable RS: Risk assessment of ritual use of oral dimethyltryptamine (DMT) and harmala alkaloids. Addiction 2007;102:24-34. 